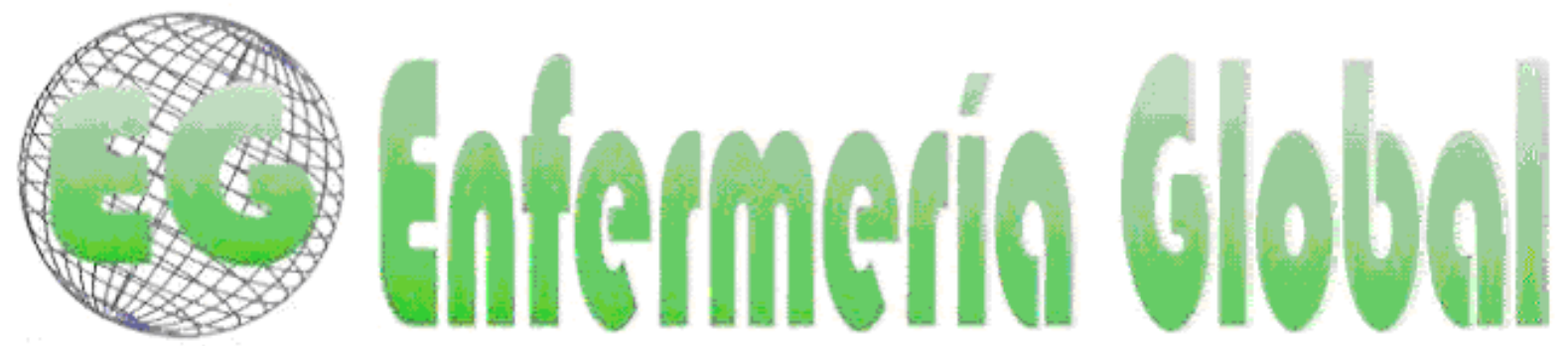

\title{
REVISIONES
}

\section{El paradigma emancipatorio y su influencia sobre el desarrollo del conocimiento en Enfermería}

The emancipatory paradigm and its influence on the development of nursing knowledge

\section{*Ramírez Elizondo, Noé Alberto ${ }^{* *}$ Quintana Zavala, Ma Olga ***Sanhueza Alvarado, Olivia ***Valenzuela Suazo, Sandra Verónica}

\author{
*Magister en Enfermería Ginecológica, Obstétrica y Perinatal. Docente del Departamento de \\ Enfermería de la Universidad de Costa Rica. E-mail: noe.ramirez81@hotmail.com **Maestra en \\ Ciencias de Enfermería. Docente del Departamento de Enfermería de la Universidad de Sonora. \\ México. ***Doctora en Enfermería. Docente del Postgrado en Enfermería. Universidad de Concepción. \\ Chile.
}

Palabras clave: Autonomía Profesional; Filosofía en Enfermería; Teoría de Enfermería Keywords: Professional Autonomy; Philosophy, Nursing; Nursing Theory

\section{RESUMEN}

El presente artículo surge por la necesidad de reflexionar sobre la influencia del paradigma emancipatorio en la Disciplina de Enfermería. Se realizó una revisión bibliográfica, en las bases de datos Scielo, Latindex, Redalyc, así como el uso de documentos escritos (libros). Se identificó que el paradigma emancipador, como medio de reflexión y crítica al poder ejercido tradicionalmente, propone medidas de participación y justicia social, arraigando importantes vestigios éticos en el accionar humano. En el caso específico de Enfermería, este proceso emancipador reorienta la visión tradicionalmente arrastrada en la esencia del ser y hacer, por lo que la apertura al pensamiento reflexivo permite modificar la manera en la cual la profesión se posiciona, brinda intervención y prepara a las (os) futuras (os) enfermeras (os).

\section{ABSTRACT}

This article arises as a need to reflect on the influence of emancipatory paradigm in the Discipline of Nursing. A literature review was conducted in the databases Scielo, Latindex, Redalyc and written documents (books). It was identified that the emancipatory paradigm as a means of reflection and critique of power traditionally exercised, proposes measures for participation and social justice, including important ethical traces in the human action. In the specific case of Nursing, this emancipatory process reorients the traditional view on the essence of being and doing, so that openness to reflective thinking to modify the way in which the profession is positioned, delivers nursing care and teach future Nurses. 


\section{INTRODUCCIÓN}

La investigación es de gran importancia para el desarrollo de la disciplina, profesión y ciencia de Enfermería, ya que a través de ella adquiere fuerza, se renueva y se torna autónoma, puesto que constituye una herramienta que permite el avance disciplinar, evita el estancamiento y conduce a dejar de lado la práctica por tradición ${ }^{(1)}$ para darle un sentido disciplinar a la práctica de enfermería.

Desde este punto de vista, resulta significativo reflexionar sobre la investigación de aspectos que revelan las particularidades del ser humano, puesto que su comprensión y análisis perfecciona la praxis, evidenciando las experiencias vividas por enfermeras(os) y usuarios(as) en sus interacciones.

Lo anterior será posible al indagar con metodologías cualitativas de investigación y utilizar paradigmas innovadores. Esta tendencia investigativa en el área de la salud, ha irrumpido con fuerza, desde aproximadamente veinte años atrás y busca, la comprensión de fenómenos complejos y cambiantes, poniendo especial interés en revelar el punto de vista del otro, del que es desconocido y no ha tenido voz, para de esta forma, construir conocimiento que incluya el sentir y la percepción de los individuos y las comunidades, en sintonía con los contextos comunitarios y permitiendo una relación dialógica con las diversas disciplinas que intervienen en las áreas de la salud, lo social y lo político ${ }^{(2)}$.

El propósito de este trabajo es reflexionar en relación a la necesidad de usar nuevos paradigmas y metodologías en el desarrollo disciplinar de Enfermería como oportunidad de fortalecer el objeto de estudio, enfatizando en el paradigma emancipatorio y su utilidad para incrementar la autonomía profesional de Enfermería, tanto en el ámbito laboral como académico.

\section{MÉTODO}

Se realizó una revisión bibliográfica en las bases de datos Scielo, Latindex, Redalyc, así como el uso de literatura escrita. El artículo se basa en un análisis referencial, que permite el desarrollo de un posicionamiento relativo a las implicaciones de la emancipación y sus alcances en el conocimiento de Enfermería. Las palabras claves para la búsqueda fueron: paradigma emancipatorio, enfermería y autonomía profesional.

\section{DESARROLLO}

\section{Paradigma y su implementación en Enfermería}

En la actualidad son utilizados diversos paradigmas en la investigación en ciencias de la salud, la exploración disciplinada en este campo se realiza principalmente dentro de dos paradigmas amplios, ambos legítimos para la investigación científica, que son el positivista y el naturalista, sin embargo, existen otros paradigmas de investigación desde las ciencias sociales que colectivamente han llegado a denominarse Teoría Crítica o Hermenéutica Dialéctica ${ }^{(3)}$. Al realizar un análisis del positivismo, postpositivismo, constructivismo y teoría crítica, los dos primeros corresponden a los paradigmas dominantes, a la perspectiva heredada, su interés es la explicación, predicción y control; mientras que el constructivismo se interesa en la comprensión, y 
reconstrucción de los hechos, así como la teoría crítica tiene un interés investigativo de reflexión, transformación, restitución y emancipación ${ }^{(4)}$.

En relación al método aplicado por cada uno de los paradigmas, el positivista se enfoca en el método cuantitativo, mientras que el postpositivismo implementa metodologías mixtas. El constructivismo y la teoría crítica utilizan predominantemente metodologías cualitativas. Sin embargo, existen perspectivas distintas donde se asevera que el uso de métodos tanto cualitativos como cuantitativos puede ser apropiado para cualquier paradigma de investigación, a pesar de que las cuestiones de método son secundarias frente a las del paradigma ${ }^{(4)}$.

La teoría crítica es un paradigma amplio, que ofrece a la Enfermería el marco de referencia adecuado para estudiar fenómenos que no son abordados con facilidad, es decir, situaciones complejas como el machismo, la violencia, la inequidad, entre otros, que por lo regular parecen ser invisibles, ya que su abordaje implica efectuar acciones equitativas y justas para mejorar la salud de las comunidades, lo cual resulta complejo desde un paradigma tradicional.

Autoras estadounidenses plantean integrar el conocimiento emancipatorio a los patrones del conocimiento de Enfermería de Carper (1978), que incluye el patrón estético o artístico, reconocido como aquello que se siente a raíz de interactuar y entender la experiencia del otro; el personal, que consiste en la forma en la que se adquiere conocimiento desde la perspectiva íntima; el ético que permite reconocer el compromiso y respeto a la vida humana y a la obligación moral de la profesión de enfermería; el patrón de conocimiento empírico, que se obtiene a través de los resultados de investigación científica y además desde su propuesta incluir; el patrón de conocimiento emancipatorio, que consiste en la capacidad no solo de notar las injusticias en un orden social, sino examinar críticamente porque las injusticias no son evidenciadas $^{(5)}$.

\section{La Teoría Crítica y el Paradigma Emancipatorio}

La teoría crítica nace con los filósofos Horkheimer y Adorno, fundadores de la Escuela de Frankfurt, quienes cuestionaban la modernidad del mundo occidental argumentando que la mitología y la tradición alimentan el proceso de alienación del propio hombre. De la tradición crítica fue Jürgen Habermans, quien liga la filosofía con la antropología y a partir de ello revela dimensiones alternativas de racionalidad comunicativa emancipadora ${ }^{(6)}$.

Habermans, es conocido como un analítico social, que centra sus estudios en la racionalidad moderna, el interés del conocimiento, la teoría de la acción comunicativa y el proceso de emancipación, señala que el conocimiento tiene carácter liberador y ético más que dominador y técnico, y los intereses constitutivos de los saberes en el ámbito técnico son las ciencias empírico - analíticas; en el ámbito práctico, la historia y hermenéutica, es decir, la interacción simbólica; mientras que el saber emancipatorio se centra en las ciencias críticas que se enfocan al estudio del poder, donde se fundamentan las ciencias sociales y filosóficas. Habermans, distingue tres racionalidades: la técnica, como el dominio de la naturaleza; la hermenéutica, como los intereses comunicativos, el propósito de la comunicación y el entendimiento entre individuos y comunidades, y la emancipatoria, que centra sus intereses en la liberación, propia de la reflexión de las disciplinas críticas ${ }^{(7)}$. 
El enfoque de la teoría crítica es la supresión de la injusticia social, la justicia se muestra como necesaria e imprescindible, el paradigma emancipatorio busca el cambio social, enfocado en la libertad y el desprendimiento, tanto en el proceso de investigación, como en el de la transformación de la sociedad misma, y cumple con tres características: incluir las experiencias del investigador en las preguntas de investigación, los objetivos de interés y las estrategias para cambiar su condición. Este paradigma abarca la distribución del poder en la situación de inequidad. En el proceso emancipatorio se habla de negociación, reciprocidad y empoderamiento, tomando a la emancipación como una posición crítica que a través del enfoque participativo lleva a una toma de conciencia, utilizando como metodología de investigación la participativa, cualitativa, por medio de un análisis crítico, permitiendo al fenómeno humano emerger y ser investigado ${ }^{(8)}$.

El interés del paradigma emancipatorio es reconocer la realidad psicológica, cognitiva, moral, político y social de todos los participantes de la investigación, incluyendo al investigador mismo, con el objetivo de centrarse en el entendimiento de la colectividad para provocar un cambio sistémico, que permita transformar la práctica y estructuras, a través del empoderamiento y el proceso transformador creando relaciones colaborativas, desarrollando estrategias de compañerismo, negociando poder y creando medio ambientes participativos, por medio de la reflexión acción, compromiso en dialogo, escuchando, realizando cuestionamiento, planteamiento de problemas y sobre todo fomentando el pensamiento crítico $^{(9)}$.

La toma de conciencia, necesaria para el desarrollo del trabajo dentro del paradigma emancipatorio ha sido entendida y promovida por Paulo Freire como un aprender a percibir las contradicciones sociales, políticas y económicas y realizar acciones contra los elementos opresivos de la realidad. "El logro de esta toma de conciencia exige, frecuentemente, la problematización de las situaciones conflictivas en que se vive, pues, de otra manera, quedarían solamente al nivel de una inconsciencia general" ${ }^{(10)}$. Lo anterior será logrado al implementar investigación acción, crítica y emancipadora, la cual persigue integrar la ciencia y la política, no para superar la resistencia al cambio entre las personas, sino para transformar esa resistencia pasiva en una oposición activa. En esta investigación el autor se integra a la comunidad no como un conocedor o un sabio sino como un igual, lo que le permite aprender de la misma y conocer realmente su sentir. "El grupo deja de ser un simple objeto de observación o de manipulación. El hombre objeto es transformado en sujeto de conocimiento y sujeto del cambio. Este paradigma pone énfasis en el contexto histórico en que se realiza la acción, se esfuerza por mantener el conocimiento dentro del escenario en que nace, para confrontarlo con la práctica social (la devolución del conocimiento), mediante tácticas y estrategias de cambio"(11).

Esta metodología en la praxis de Enfermería permite desarrollar la observación comprensiva de los fenómenos, donde se establece no sólo una relación de simpatía entre el (la) investigador(a) y los miembros de la comunidad, sino que crea un análisis en conjunto de las principales problemáticas identificadas por aquellos(as) que las vivencian, para establecer estrategias e intervenciones colectivas orientadas a la solución participativa del problema(s). La introducción del conocimiento emancipatorio a la enfermería es el camino para hacer posible el cambio social y estructural y es hora de examinar con respecto a la critica las influencias sociales sobre el bienestar y la salud. El conocimiento emancipatorio como la capacidad no solo de evidenciar injusticias en un orden social, sino también examinar críticamente porque las injusticias parecen no ser notadas o permanecer invisibles y permite identificar 
cambios sociales y estructurales necesarios para corregir los males sociales institucionales ${ }^{(5)}$.

Las(os) Enfermeras(os) necesitan crear una práctica en la que se pueda continuar desarrollando el conocimiento que va más allá del desarrollo de habilidades para resolver problemas. Esta praxis se denomina, "el proceso del conocimiento emancipatorio", lo que obliga a ampliar los marcos de referencia y detectar patrones y estructuras en las situaciones que son problemáticas del día a día ${ }^{(5)}$.

\section{La autonomía de Enfermería como un proceso emancipatorio}

La disciplina de Enfermería, al igual que la mayoría de áreas de la salud, se mantiene en un continuo avance en el cuerpo de conocimientos que le permita orientar sus esfuerzos a desarrollar una profesión con bases sólidas y posturas críticas ante las transformaciones sociales que se dan en la mayoría de países del orbe. Es por medio de dichos cambios que la profesión ha avanzado de manera continua a la formulación de interrogantes relacionadas con su papel (hacer) y esencia (ser) y postular así direccionamientos óptimos que le permitan una mejora de la posición social actual, enrumbando los esfuerzos por una autonomía que rescata un enfoque de mayor equidad e igualdad en el ámbito sanitario, ante el poder patriarcal de otras disciplinas que se imponen.

"En estos tiempos que vivenciamos un mundo globalizado, el debate pluralista desde donde la Enfermería va creciendo como disciplina, debe abordar dos aspectos: por una parte, el mundo complejo en que se encuentra inmersa la profesión de Enfermería y, por otro, el reconocimiento de las condiciones de inequidad que afectan a una parte importante de las mujeres. La Enfermería es aún una profesión en la que sus integrantes son mayoritariamente mujeres, que habitan en un sistema de inequidad y cuyas experiencias vivenciales las sitúan en un contexto particular cuando se enfrentan a problemáticas sociales y sanitarias, ya que el sistema patriarcal les encomienda el aspecto de cuidado, que en nuestro mundo está reconocidamente feminizado" ${ }^{\prime(12)}$.

Es preciso retomar la evolución histórica de Enfermería, entendida ésta no sólo en su proceso de conformación cognitiva, sino también en el grupo particularmente femenino que le conforma, donde es necesario aclarar que por ser así no le responsabiliza de errores cometidos, sino más bien se alude a una sociedad meramente patriarcal que por tradición ha arremetido en contra de la mujer, y de una $\mathrm{u}$ otra forma le ha asignado papeles de inferioridad y de carácter punitivo, donde el hombre "Dios" dominante mantiene el poder controlador sobre sus ángeles (véanse estos como enfermeras), por tanto, la continuidad de un paradigma de acceso al poder y al saber, centrado en el arquetipo viril de un modelo de hombre racional (toda una simbología del dominador) ha dado lugar al ocultamiento de formas de dominio que perviven al margen de la crítica y la acción liberadora, lo que da pie a una discriminación histórica sobre aquellos(as) menos favorecidos(as), como las mujeres y otras categorías socio-demográficas que padecen formas específicas de dominación. "Dichas formas de dominio, potenciadas en la civilización (y la barbarie) capitalista, son mucho más que un efecto superestructural o un reflejo de las condiciones económicas. Enhebran el tejido social y han penetrado en la psiquis y la cultura humana. No de otra manera se explica la permanencia de patrones racistas, sexistas y relaciones patriarcales autoritarias que irradian el tejido social a las puertas del nuevo milenio, incluso bajo el manto de discursos pretendidamente democráticos o antisistema"(13). 
La historia demuestra que Enfermería ha dado saltos importantes en el posicionamiento filosófico sobre el cuerpo de conocimientos que le sustentan y el uso de los mismos en la práctica cotidiana, en los múltiples sectores en los que se interviene, por tanto, es necesario un camino a un dominio de igualdad y equidad, que le facilite ser copartícipe ${ }^{(14)}$ de forma igualitaria, en las tomas de decisiones referentes a salud, viendo esta última como una acción dirigida hacia una persona o una colectividad.

Tal libertad o independencia representa una ruptura del poder ejercido por diversos determinantes sociales que tiene que ver con un constructo establecido entre el ser y el hacer (sin dejar de lado el antecedente histórico), que de una forma llevó a un estancamiento de la disciplina, pero al mismo tiempo le permitió recapitular los rumbos del futuro.

Si bien es cierto, la esencia de la profesión durante muchos años estuvo matizada por una doblegación y sumisión a modelos positivistas biomédicos, el discurso referente a la evolución no debe estancarse en el antecedente, sino más bien evolucionar a la reflexión crítica, "es así que el dominio de la naturaleza se convierte en un dominio de los seres humanos sobre otros seres humanos, y en último lugar una pesadilla de autodominio"(15).

Es posible que el poder movilice al ser humano o una disciplina a entablar discursos dicotómicos de la realidad y lo deseado, o por el contrario dé lo justo por lo demostrado, sin embargo, como se mencionó anteriormente, el estancamiento de la disciplina no es exclusivo de sus integrantes, sino que involucra diversos determinantes que hacen uso del poder para generar beneficio o perjuicio.

¿Será acaso que Enfermería ha sido de una u otra forma obstaculizada por el discurso de apropiación de otros, e incluso el propio?, entendido este como el derecho a hablar en nombre de ciertos discursos, comprenderlo y servirse del mismo, tomando en cuenta que para poder accesarlo se debe ser parte de un grupo monopolizante regido por ciertos sujetos o instituciones, como "el discurso en el siglo XVI no constituía un discurso común de libre circulación sino que quedaba circunscrito a un ámbito muy reducido de eruditos al igual que el discurso médico, y de algún modo el literario"(16), condicionando así una equidad más que justa en la participación de la mayoría.

¿Es acaso el discurso de Enfermería poco convincente, o más bien opacado por grupos de poder social dentro del sector salud?, y si es así, ¿hacia dónde ha evolucionado la apropiación del mismo?

"Por consiguiente la cuestión fundamental que debemos analizar es si ser un ser humano es estar determinado por un lenguaje y por un esquema conceptual de concretos, es decir, habitar siempre en un sistema de pensamiento que inhabilita para pensar o hablar desde un punto de vista idealmente neutral al ambiente. Ésta creo, sería la posibilidad de que en cualquier momento, en cualquier situación, cualquier ser humano pueda interpelar alrededor suyo con la intención de deslegitimar lo establecido" ${ }^{(17)}$.

Si bien es cierto no se intenta refutar lo construido históricamente (por no decir estancado), sí se hace hincapié a lo que se debe aún hacer, libre de lo que siempre se ha hecho bajo el discurso y tradición impuesto, lo que brinda la posibilidad de 
analizar si lo que en la actualidad se posee como profesión es un valor dado por otros, y por ende asumido por nosotros.

Bajo esta reflexión, la disciplina de Enfermería ha generado un conmocionado cambio en las estructuras de ver la intervención y posicionarse de manera autónoma como disciplina en el sector salud, siendo ese el gran aporte del proceso emancipatorio que los(as) profesionales han generado en las últimas décadas, la búsqueda continua del por qué Enfermería posee un vasto cuerpo de conocimientos que sustentan científicamente la práctica, que "contamos con la lucidez necesaria para cuestionar lo que tenemos y para decidir lo que aspiramos. Estas reflexiones nos retrotraen de nuevo a la hipótesis de que el ser humano no es alguien absolutamente ignorante, sino que de algún modo ha de conocer y reconocer el ideal, en el sentido menos de representarse en algún momento lo que quiere llegar a ser"(17).

\section{Aplicación de la emancipación al ámbito laboral de Enfermería}

Enfermería ha sido partícipe de la atención patocéntrica, centrada en el poder de los conocedores (sabios del área), con direccionamientos rígidos sobre las decisiones de aquellos(as) con menos saber (usuarios(as)), bajo lineamientos positivistas (biologisistas) dominados por un paradigma categórico, lejos de la sensibilidad y realidad de quienes se intervienen.

Es a través del análisis de situaciones como la anteriormente descrita, que se ha intentado modificar los modelos de atención de Enfermería enfocados a condiciones más justas y de interacción, con una gran responsabilidad en la participación de los diversos sectores sociales que involucra un constructo colectivo, y que de una u otra forma afectan de manera negativa o positiva los resultados de las condiciones de salud de las personas.

Esta participación necesariamente acarrea una redistribución del poder y recursos entre aquellos que toman las decisiones en salud, ya que si se abren espacios de participación social el personal sanitario pierde su hegemonía dominante en la resolución de necesidades en salud y pasa a ser un consenso colectivo. Desde este concepto se visualiza a la persona como un(a) receptor(a) de los servicios y se espera opiniones sobre la atención recibida y una participación compartida en lo que a condiciones de salud se refiere ${ }^{(18)}$, eliminando así el marcado modelo de atención "centrado en la enfermedad, práctica individual, hegemonía de la atención hospitalaria y el uso intenso de tecnologías incapaces de intervenir eficazmente sobre una parte importante de los determinantes de la mortalidad, la incapacidad y la enfermedad" (19), involucrando el abordaje de los diversos determinantes sociales de la salud, y un papel activo de las instancias comunitarias.

Esto implica la activación por parte de Enfermería de procesos de acción e interacción compartida con los(as) usuarios(as), una mayor delegación de responsabilidad a los (as) mismos(as) en la toma de decisiones, dentro de los diversos ámbitos que se desempeña los(as) profesionales en Enfermería.

Para mejorar la participación social a nivel asistencial se plantea que primero debe haber puertas de entrada a los sistemas de salud, donde el (la) ciudadano(a) pueda tener implicaciones en la toma de decisiones ${ }^{(19)}$. Otro elemento es el acceso a la información, tomando este como los mecanismos para que la población se encuentre enterada de los servicios que se brindan, sus derechos, precios y calidad de los 
ofrecidos. Se debe tomar en cuenta que las personas sólo serán participes si consideran que sus acciones influyen en los resultados y por ende se logrará un beneficio.

En el caso de Enfermería, lo anterior involucra un actuar ético, donde producto de la justicia y autonomía, se tome en cuenta en todo momento la opinión y participación activa de los(as) beneficiarios del servicio, entendiéndose la participación como un modo de intervención que en conjunto con las personas conducen a estrategias para mejorar la salud de los individuos, recuperarse de la enfermedad o evitar llegar a ésta.

\section{Currículos de Enfermería bajo un enfoque emancipatorio}

"Se puede observar que los nuevos roles de la política en el acontecer de América Latina, se proponen la reconstrucción del poder político desde las praxis ciudadanas participativas y liberarias. Más allá de la ideologización hegemónica de las clases y los sectores de poder, se requiere una sociedad que en efecto reconozca la pluralidad y diversidad de su tejido social, como principio de avance para programas de ciudadanía que permitan asumir y consensuar diferencias"(14), por lo que generar o desarrollar fuerzas de producción posibilita reducir la presión del marco institucional y cambiar la base de una obediencia hacia la cultura, a una base racional ${ }^{(20)}$, condición que no es exclusiva del proceso de formación educativo de los individuos, pero sí altamente relevante en la preparación a nivel universitario, tomando en cuenta que "el hombre al tener una visión de su praxis educativa sobre lo real sirve a los propósitos de la dominación intelectual, sin poder, o puede desplegar una acción emancipatoria en el contexto académico"(21), por tanto, la educación cumple un eje fundamental en el proceso de formación intelectual y actitudinal de los individuos que asisten a instancias de Educación Superior, ya que la Universidad debería responder a la contextualización de los sistemas sociales, orientando sus propuestas educativas hacia las necesidades actuales de los individuos, con currículos que por medio de "revisiones o cambios que determinan un perfil de formación y asignación en la estructura social. En este sentido se construye como espacio político educativo y no meramente técnico pedagógico, por ejemplo, las decisiones acerca del modo de organización de los contenidos 0 de la estrategia de enseñanza - si bien son técnicas- tienen un sentido político, involucran más allá de lo metodológico un posicionamiento social"(22).

Añorga, citada por Amaro, argumenta que todo plan curricular se compone de varias dimensiones: "la dimensión filosófica (define los ideales propósitos y metas que orientan las tareas formativas del proceso educativo), social (ubica el currículo dentro de la realidad nacional e internacional), psicopedagógica (reconoce la integralidad del ser humano y la necesidad de la reflexión y el diálogo), metodológica (orienta al camino viable para los aprendizajes y caracteriza la interacción entre docentes y estudiantes, y entre ellos y su entorno), tecnológica (define los medios y procesos a utilizar para lograr los fines y objetivos de la universidad y el proceso educativo), y comunicacional (considera la educación como sistema de influencias que tiene como fin la socialización de las personas, a partir de intereses y valores de una sociedad, en un contexto determinado, que permite compartir emociones, interrogantes y reflexiones, desarrollar capacidad de pensamiento lógico y organizar acciones para transformar la realidad)" (23).

Los currículos de Enfermería, desde la teoría crítica podrían guiar al reencuentro pedagógico discursivo manteniendo ideas emancipatorias del sujeto trascendental, la relación entre objetividad y racionalidad, la no coincidencia entre el ser y la razón, así 
como la razón y su principio opuesto(21). "Así mismo, el reencuentro pedagógico discursivo no significa el fin de la historia, significa la búsqueda de cómo desplegaron los meta-relatos de dominación en la relación docente-alumno, y a cuál racionalidad obedecieron"(21), siendo este el verdadero reto para la conformación de modelos educativos de Enfermería, que eliminen el patrón dominante del profesorado (yo conocedor), ejercido tradicionalmente hacia el estudiantado (el no conocedor), como medio de "violencia hacia sus semejantes en formación con el afán de mantener un estándar profesional y de llegar al reconocimiento que tanto ansían, en el contexto de un mercado competitivo y voraz, y de un ejercicio en conflicto de roles multifactoriales"(24).

Por tanto, "la Enfermería requiere de docentes altamente calificados para promover el aprendizaje autónomo de sus estudiantes, el mismo que dará origen a egresados comprometidos con su autoeducación, de tal forma que como grupo profesional asuman un papel protagónico en el desarrollo de la humanidad, y aprendan desde el aula a crear y participar en un mundo más humano, equitativo y responsable"(25).

La problemática radica que en la actualidad la mayoría de los currículos de Enfermería poseen una estructura que consiste en una serie de pasos que inician desde las ciencias básicas, hasta la práctica, lo que a su vez acarrea una jerarquía de roles de los que poseen el conocimiento, conduciendo a una subordinación de los que están más cercanos en la práctica, para con los que se encuentran en la producción del conocimiento ${ }^{(26)}$, por lo que puede apreciarse que la distribución cuantitativa de la mayoría de los currículos de Enfermería están enfocados a la preparación de personas bajo un enfoque principalmente asistencial ${ }^{(27)}$, y con bajo soporte investigativo a causa del recargo asignado a las diversas prácticas mecanicistas.

Es así que en América Latina, la transformación “... no puede limitarse a recurrir a modos de investigación comúnmente aceptados como hizo en casos del interés técnico y del interés práctico. Aquí no basta con sondear los fundamentos de disciplinas establecidas, sino que hay que embarcarse de reflexiones epistemológicas a fuer de propedéuticas para formular una nueva concepción de investigación social y de investigación filosófica"(20).

Igualmente, en los currículos de Enfermería la investigación permite la formulación de respuestas a diferentes problemáticas de índole social, que por medio del uso de estrategias reflexivas y de análisis crítico permea la sensibilidad de aquellos(as) que desean conocer sobre lo estudiado. En este sentido el uso de estrategias cualitativas, enfocadas a la competencia cultural como conocimiento aplicado a la Enfermería será uno de los temas de investigación más significativa en los próximos años, condición que normalmente la investigación cuantitativa no ha tenido en estos aspectos ${ }^{(28)}$, sin embargo, más que un alejamiento de ambos posicionamientos (cuantitativocualitativo), debe implementarse una complementariedad entre ambos, y mejorar así las respuestas ante las interrogantes surgidas.

Lo que sí es necesario, es la comprensión a la que conduce la emancipación en lo referente a investigación, ya que el abordaje se orienta más a la realidad de las personas, generando un acercamiento que permite identificar las realidades de los(as) que las viven, aspectos necesariamente involucrados en la formación de profesionales en Enfermería, que "faculten a utilizar y desarrollar recursos de aprendizaje concordantes a los requerimientos actuales de la sociedad, aprovechando 
la actividad científico tecnológica y empleando medios existentes en toda su potencialidad. Lo que involucra la creación de ambientes propicios al crecimiento individual de los alumnos y al desenvolvimiento académico, favoreciendo una formación profesional activa, creativa y cuestionadora"(29).

La investigación cualitativa (sin minimizar la importancia cuantitativa) representa una adecuada herramienta para alcanzar respuestas certeras a un ideal de participación activa por parte de la persona que investiga y aquellos(as) partícipes de la misma, siendo dentro de ésta la investigación acción una manera de "poner de manifiesto que ciertas distancias existentes entre la teoría y la práctica no pueden superarse mientras no se modifiquen los factores contextuales. En este caso, la investigación acción puede llevar desde la reflexión sobre las estrategias políticas que conduzcan a la modificación del "sistema" de modo que sea posible la acción educativa"(30).

La articulación entre un currículo que le permita a los(as) estudiantes el desarrollo de un pensamiento crítico y reflexivo, basado en la importancia de establecer estrategias que generen el análisis de problemáticas reales, junto con la enseñanza de modelos de investigación bajo el mismo enfoque, consolida el compromiso que Enfermería durante mucho tiempo ha asumido con la sociedad: la conformación de estrategias sanitarias que favorezcan a las personas a tomar decisiones respecto a su propia salud, y una comprensión multidimensional de éstas.

\section{CONCLUSIONES}

El paradigma emancipador, como medio de reflexión y crítica al poder ejercido tradicionalmente, propone medidas de participación social y justicia, arraigando importantes vestigios éticos en el accionar humano. En el caso específico de Enfermería, este proceso emancipador reorienta la visión tradicionalmente arrastrada en la esencia del ser y hacer, por lo que la apertura al pensamiento reflexivo permitió modificar la manera en la cual la profesión se posiciona frente a su rol, brinda intervención y prepara las(os) futuras(os) enfermeras(os).

La Autonomía en Enfermería como medio de independencia disciplinar, representa un importante paso, producto del análisis de aquellas(os) enfermeras(os) que reflexionaron ante los modelos dominantes provenientes del patriarcalismo y positivismo tradicional, favoreciendo de manera justa un accionar fundamentado en el crecimiento científico que la profesión ha venido evolucionando en los últimos años. Es así que la autonomía de Enfermería no es más que respuesta sustentada ante modelos sociales ejercidos tradicionalmente por estructuras dominantes del poder, justificadas en el conocimiento absoluto de sólo unos cuantos.

Es producto del proceso del análisis reflexivo de lo que Enfermería es y hace, que surgió también la necesidad de modificar la manera en la cual se brinda asistencia a las poblaciones o individuos que requieren de los servicios sanitarios, enrumbando el pensamiento hacia la ruptura de enfermeras(os) dominantes del saber frente a individuos carentes del mismo, y formular así mecanismos sanitarios donde los(as) usuarios(as) representan entes activos de su propia salud, por ende la intervención se convierte de modelos reduccionistas a modelos abiertos y de interacción social.

Los logros en la disciplina de Enfermería producto de la emancipación como medio reflexivo, también matizan el ámbito académico, al sustentar los currículos con estrategias de aprendizaje que le faciliten al estudiantado un análisis crítico ante diversas problemáticas abordadas, generando un conocimiento compartido desde los 
saberes individuales a los saberes colectivos, y donde el docente representa un acompañante durante el proceso. De igual manera, el posicionamiento emancipador ha permitido orientar la investigación desde la academia bajo modelos menos cuánticos del ser humano, y más bien ha generado apertura hacia el acercamiento y participación de aquellos(as) que poseen una problemática dada y que necesariamente debe ser abordada.

\section{REFERENCIAS}

1.- Durán de Villalobos, M. La ciencia, la ética y el arte de enfermería a partir del conocimiento personal. Rev Aquichan. 2005; 5(1): 86-95.

2.- De la Cuesta, B. La investigación cualitativa y el desarrollo del conocimiento de enfermería. Texto Contexto Enferm, 2010; 19(4): 762-6.

3.- Polit D, Hungler B. Investigación Científica en Ciencias de la Salud. (6 $6^{\text {ta }} \mathrm{Ed}$ ). México: Mc Graw-Hill; 2000.

4.- Guba E, Lincol Y. Paradigmas en competencia en la investigación cualitativa. Denman C, Haro J.(comps.). Por los rincones. Antología de métodos cualitativos en la investigación social. El Colegio de Sonora. Hermosillo, Sonora, México. 2002; 113-45. 5. - Chinn P , Kramer M. Integrated Theory and Knowledge Development in Nursing. $7^{\text {th }}$ Edicion. USA: Mosby. Citado por Polchert J. (2008) The leading Edge. Delta Gamma Chapter of Sigma Theta Tae Internacional at Marquette University. Winter Edition, [en línea]. Recuperado el 1 de Julio del 2011, de: http://www.deltagammastti.org/docs/leadingedge0108.pdf

6.- Carretero A. Jürgen Habermans y la primera Teoría Crítica: encuentros y desencuentros. Cinta de Moebio. 2006; 27:11-26.

7.- Villalobos M. Ciencia Social como Crítica Social. La revolución epistemológica de Karl Max. $1^{\text {era }}$ Ed. Santiago de Chile: LOM; 2010.

8.- Del Valle N. Justicia, teología y teoría crítica en la obra tardía de Max Horkheimer. Alpha Osorno. 2010; 31:55-67.

9. - King B. (cb.king.atverizon.net) Mertens and Emancipatory Paradig. 2004. Correo electrónico enviado a: gdemetrionatmsn.com. Recuperado el 28 de Junio del 2011, de: http://lists.literacytent.org/pipermail/aaace-nla/2004/001214.html

10.- Martínez M. Epistemología Feminista y Postmodernidad. Cinta de Moebio. 2003; 16: sin número de páginas.

11.- Vejarano M. Comp. La investigación participativa en América Latina. Antología. [internet]. Pátzcuaro, Michoacán, México: Biblioteca Digital; 1983. [Consultado el 27 de Junio del 2011] Disponible en: http://tariacuri.crefal.edu.mx/crefal/crefal2011/

12.- Valdés G. Hacia un nuevo paradigma de articulación (no tramposo) de las demandas emancipatorias. Utopía y praxis latinoamericana. 2001; 6 (14):48-57.

13.- Díaz Z, Márquez, A. Características emancipadoras de los nuevos roles de la política en América Latina. 2009; 24 (24): 163-78.

14.- Habermas J. Habermas y la modernidad. Cátedra. Colección Teorema. 4ta. Edición. Madrid: España; 1999.

15.- Albano A. Michael Focaoult. Glosario epistemológico. Editorial Quadrata. Buenos Aires: Argentina; 2007.

16.- Miller N, Salazar R, Valdés G. Paradigmas emancipatorios y movimientos sociales en América Latina. Grupo América Latina: Filosofía social y Axiología (GALFISA) del instituto de filosofía de la Habana; 2006.

17.- Vásquez M, Siqueira E, Kruze I, Da Silva A, Leite I. Los procesos de reforma y la participación social en salud en América Latina. Gaceta Sanitaria. 2002; 16 (1): 30-8. 
18.- Martín M, Ponte $C$, Sánchez M. Participación social y orientación comunitaria en los servicios de salud. Gac Sanit. 2006; 20 (1): 192-202.

19.- Mc Carthy, T. La teoría Crítica de Jurgen Habermas. 4ta. Edición. España: Editorial Tecno; 2002.

20.- Calzadilla P. La Universidad y la emancipación histórica del sujeto racional en la ética de la posmodernidad. Investigación y Posgrado. 2006; 21 (2): 269-86.

21.- De Stefano O. Apuntes para innovaciones curriculares en el nivel universitario. Universidades. 2004; 27: 15-19.

23.- Amaro M. Un nuevo paradigma para la Universidad Nueva. Revista Cubana Habanera de Ciencias Médicas. 2010; 9 (1): 116-26.

24.- Ayala-Valenzuela R. Biiopoder: el poder y la violencia en la formación de profesionales de enfermería. Educación Médica. 2008; 11 (3):125-129.

25.- Cerezo A, Hernández J. Formando docentes. Una experiencia desde el constructivismo. Aquichan. 2008; 8 (1): 64-73.

26.- Medina J, Castillo S. La enseñanza de la Enfermería como una práctica reflexiva. Texto Contexto Enferm. 2006; 15 (2):303-11.

27. - López J, Sánchez L. Aproximación reflexiva y analítica sobre la formación permanente para docentes de la disciplina enfermera. Educare 21: enseñando a cuidar. Sin año; 1 -13.

28.- Lillo C, Vizcaya M, Domínguez S, Galao M. Metodología cualitativa.

Investigación cualitativa en enfermería y competencia cultural. Index de Enfermería. 2004; 13 (44-45):47-50.

29.- Arriata A. La innovación en la educación superior de enfermería y los aportes del diseño de instrucción. Rev. Latino-am.enfermegem. 1999; 7 (2): 5-13.

30.- Elliot J. La investigación acción en educación. 5ta. Edición. Editorial Morata. Madrid; 2005. 